\title{
Correcting for Background Noise Improves Phenotype Prediction from Human Gut Microbiome Data
}

Leah Briscoe $^{1 *}$, Brunilda Balliu ${ }^{2}$, Sriram Sankararaman ${ }^{3,4,5}$, Eran Halperin ${ }^{3,4,5,6,7 *}$, Nandita R. Garud $^{4,8 *}$

1. Bioinformatics Interdepartmental Program, University of California Los Angeles, Los Angeles, Los Angeles, CA, 90095, USA

2. Department of Computational Medicine, University of California Los Angeles, Los Angeles, CA, 90095, USA

3. Department of Computer Science, University of California Los Angeles, Los Angeles, CA, 90095, USA

4. Department of Human Genetics, University of California Los Angeles, Los Angeles, CA, 90095, USA

5. Department of Computational Medicine, David Geffen School of Medicine, University of California Los Angeles, Los Angeles, CA, 90095, USA

6. Department of Anesthesiology and Perioperative Medicine, David Geffen School of Medicine, University of California Los Angeles, Los Angeles, CA, 90095, USA

7. Institute of Precision Health, University of California Los Angeles, CA, 90095, USA

8. Department of Ecology and Evolutionary Biology, University of California Los Angeles, CA, 90095, USA

*Co-corresponding authors. Correspondence should be addressed to: leah.briscoe@ucla.edu, ehalperin@cs.ucla.edu, and ngarud@ucla.edu

\section{Abstract}

The ability to predict human phenotypes accurately from metagenomic data is crucial for developing biomarkers and therapeutics for diseases. However, metagenomic data is commonly affected by technical or biological variables, unrelated to the phenotype of interest, such as sequencing protocol or host sex, which can greatly reduce or, when correlated to the phenotype of interest, inflate prediction accuracy. We perform a comparative analysis of the ability of different data transformations and existing supervised and unsupervised methods to correct microbiome data for background noise. We find that supervised methods are limited because they cannot account for unmeasured sources of variation. In addition, we observe that unsupervised approaches are often superior in addressing these issues, but existing methods developed for other 'omic data types, e.g., gene expression and methylation, are restricted by 
parametric assumptions unsuitable for microbiome data, which is typically compositional, highly

skewed, and sparse. We show that application of the centered log-ratio transformation prior to

correction with unsupervised approaches improves prediction accuracy for many phenotypes

while simultaneously reducing variance due to unwanted sources of variation. As new and larger essential for generating reproducible microbiome analyses.

\section{Introduction}

The human gut microbiome is associated with a number of host phenotypes including

47 colorectal cancer ${ }^{1}$, obesity ${ }^{2,3}$, and antibiotic consumption ${ }^{4-7}$, among other traits ${ }^{8,9}$. Despite the

48 promise of the microbiome as a diagnostic tool, significant challenges remain for accurate

49 prediction of human phenotypes from microbiome data. One major challenge is that several

50 covariates are known to introduce unwanted variation and systematically bias the relative

51 abundances of taxonomic units in microbiome samples ${ }^{10-16}$. These covariates include host diet

52 and sex ${ }^{17-19}$, sample storage $\mathrm{e}^{20}$, cell lysis protocol ${ }^{10,21}$, DNA preservation and storage protocol ${ }^{22}$,

53 preparation $\mathrm{kit}^{23}$, extraction method ${ }^{24,25}$, and primer choice ${ }^{21}$. This variation can substantially

54 reduce the ability to predict host phenotypes from microbiome data. In addition, when these

55 variables are also correlated with the phenotype of interest, they can act as confounders, inducing

56 associations between the microbiome and the phenotype and artificially inflating phenotypic

57 prediction accuracy. For example, confounding covariates were found to be pervasive ${ }^{26}$ in one of

58 the largest metagenomic datasets available, the American Gut Project (AGP) ${ }^{27}$. Confounding can

59 also arise when datasets from different studies are combined together to augment the power to

60 detect associations ${ }^{8,28}$, a practice that is becoming increasingly common ${ }^{29-33}$ and is potentially a

61 powerful means to validate results in a discovery dataset with held out datasets ${ }^{1,34,35}$. For 
example, Gibbons et al. ${ }^{36}$ found that combining datasets to detect members of the microbiome that were associated with colorectal cancer resulted in false positive detection of differentially abundant taxa due to the different case-control ratio in different combined studies.

66 a dearth of methods specially equipped for removing unwanted variation in microbiome data.

67 Existing methods repurposed from other domains, including gene expression ${ }^{39,40}$ and methylation ${ }^{41,4237-39}$, generally fall into two categories: supervised methods, where the sources of variation must be explicitly specified, and unsupervised methods, where the sources of variation are first inferred and then removed before association or prediction. The most popular supervised

71 methods are batch mean centering $(\mathrm{BMC})^{43}$, which centers data batch by batch, and ComBat ${ }^{44}$

72 and limma ${ }^{45}$, which both use empirical Bayes. However, since many sources of variation may be

73 unknown, and moreover, the extent of variation they introduce may vary from dataset to

74 dataset $^{13,36,40-42}$, unsupervised approaches ${ }^{43-45}$ for covariate correction may be more effective in 75 removing background noise.

Despite their promise, the repurposed supervised and unsupervised approaches ${ }^{43-45}$ are

77 not suitable for microbiome data because they rely on assumptions that the data is normally

78 distributed. However, taxonomic features are often sparse ${ }^{46,47}$ due to taxa having abundances

79 below the detection limit of sequencing ${ }^{46}$, or taxa being absent in certain samples, resulting in

80 skewed non-normal distributions. Additionally, microbiome data is compositional, or, in other

81 words, typically represented as a relative abundance table in which the frequencies of taxonomic

82 units within a sample sum to one. This induces correlations between taxonomic units within a

83 sample. Recently, supervised methods were proposed explicitly for microbiome data, and include

84 percentile normalization ${ }^{31}$, Partial Least Squares Discriminant Analysis ${ }^{48}$, and multiplicative bias 
85 correction $^{15}$. Both percentile normalization ${ }^{31}$ and Partial Least Squares Discriminant Analysis ${ }^{48}$

86 aim to find predictive features in fully labeled data with known batches and known phenotypes,

87 and are not designed for prediction of phenotypes in unlabeled data, while multiplicative bias

88 correction $^{15}$ requires a reference sample in which the species abundance distribution is known,

89 and thus cannot be applied to the majority of microbiome data that lack such references. Given

90 that these methods are supervised and thus cannot be applied to unlabeled data, there still

91 remains a need in the microbiome field for unsupervised approaches that can adjust for both

92 measured and unmeasured variables.

93 In this paper, we evaluated different supervised background noise correction

94 approaches ${ }^{49-51}$ that are commonly used for microbiome data and compared them to an

95 unsupervised approach in which the top principal components (PCs) from microbiome data are

96 regressed from OTUs or $k$-mers (a reference-agnostic feature alternative to OTUs, based on short

97 substrings of length $k$ in raw sequences) after application of the centered log ratio (CLR). This

98 PC correction approach has been used in other applications, such as correcting for population

99 structure in genetic data ${ }^{70-72}$ and morphological differences in a study population of

100 sticklebacks ${ }^{73}$, but to date has not been applied to microbiome data. CLR is widely used for

101 compositional data and specifically in microbiome contexts ${ }^{28,52-57}$, and is a suggested

102 transformation prior to factor analysis, such as principal components analysis (PCA), because it

103 breaks the dependence between features while also ensuring that the resulting covariance matrix

104 is non-singular ${ }^{53}$. CLR additionally makes data more normally distributed ${ }^{48}$. In this paper, we

105 show that CLR transformation is a crucial step in revealing sources of background noise for

106 proper covariate correction and phenotype prediction. We found that the combined approach of

107 PC correction in conjunction with CLR can simultaneously address the compositionality and 
108 non-normality, while use of $k$-mers addresses sparsity, of microbiome data all while improving

109 the ability to predict human phenotypes after correcting for background noise.

110 Throughout this study, we highlight important considerations for phenotype prediction

111 from large cohort and cross-study analyses, which we hope paves the way for higher

112 reproducibility across microbiome studies.

113

114 Results

115 We analyzed three metagenomic datasets for evidence of biological and technical

116 covariates that could introduce noise or confounding that, as a result, could diminish or inflate

117 phenotype prediction accuracy. We evaluated the ability of three popular supervised approaches

118 for microbiome data ${ }^{49-51}$ and an unsupervised approach, PCA correction, to correct for noise and

119 confounding in these three datasets, and the impact of this correction on phenotype prediction

120 accuracy. We focused on three phenotypes of interest: body mass index (BMI), antibiotic

121 consumption, and colorectal cancer (CRC) (Table 1). The datasets we analyzed included: (i) the

122 American Gut Project $^{27}$ (AGP), which has known confounding variables ${ }^{26}$ (ii) a pooled dataset

123 composed of three $16 \mathrm{~S}$ datasets with healthy and $\mathrm{CRC}$ individuals (hereafter referred to as

124 'CRC-16S' $)^{31}$, and (iii) a pooled dataset composed of seven whole genome sequenced datasets

125 (WGS) with healthy and CRC individuals (hereafter referred to as 'CRC-WGS')1. These datasets

126 allowed us to assess noise and confounding both within a dataset (AGP) and across pooled

127 datasets (CRC-16S and CRC-WGS). 


\begin{tabular}{|c|c|c|c|c|c|}
\hline Phenotype & Joined dataset & $\begin{array}{c}\text { Number of } \\
\text { samples }\end{array}$ & $\begin{array}{c}\text { Number of } \\
\text { studies }\end{array}$ & $\begin{array}{c}\text { Sequencing } \\
\text { method }\end{array}$ & $\begin{array}{c}\text { Published } \\
\text { Sources }\end{array}$ \\
\hline $\begin{array}{c}\text { Body mass } \\
\text { index }\end{array}$ & $\begin{array}{c}\text { American Gut } \\
\text { Project (AGP) }\end{array}$ & 16,225 & $\begin{array}{c}1 \text { (multiple } \\
\text { sequencing } \\
\text { batches) }\end{array}$ & $16 S$ & $\begin{array}{c}\text { McDonald et } \\
\text { al. }\end{array}$ \\
\hline $\begin{array}{c}\text { Antibiotic } \\
\text { history }\end{array}$ & $\begin{array}{c}\text { American Gut } \\
\text { Project (AGP) }\end{array}$ & 21,862 & $\begin{array}{c}1 \text { (multiple } \\
\text { sequencing } \\
\text { batches) }\end{array}$ & $16 S$ & $\begin{array}{c}\text { McDonald et } \\
\text { al. }\end{array}$ \\
\hline $\begin{array}{c}\text { Colorectal } \\
\text { Cancer }\end{array}$ & CRC-16S & 574 & 3 & $16 S$ & $\begin{array}{c}\text { Baxter et al. } \\
\text { Zeller et al. } \\
\text { Zackular et al. }\end{array}$ \\
\hline $\begin{array}{c}\text { Colorectal } \\
\text { Cancer }\end{array}$ & CRC-WGS & 813 & 7 & WGS & $\begin{array}{c}\text { Feng et al. } \\
\text { Yu et al. } \\
\text { Vogtman et al. } \\
\text { Hannigan et al. } \\
\text { Thomas et al. } \\
\text { Zeller et al. }\end{array}$ \\
\hline
\end{tabular}

130 Table 1._Datasets used in this study. Two pooled datasets composed of multiple studies are 131 abbreviated as CRC-16S ${ }^{58-60}$ and CRC-WGS ${ }^{1,59,61-64}$, whereas the American Gut Project

$132(\mathrm{AGP})^{27}$ is composed from one source study but has several known confounders ${ }^{26}$.

134 Background noise due to technical and biological variables is widespread in microbiome 135 data

136 While it is known that there are biases and confounders in microbiome data ${ }^{26}$, it is not

137 well understood how these factors impact our ability to predict host phenotypes from

138 metagenomic data. To assess the impact of biological and technical contributors on microbiome

139 variability and microbiome-phenotype relationship, we performed PCA on CLR-transformed

140 (see Methods) OTUs and $k$-mers derived from the raw metagenomic reads. We observed that the

141 top two PCs separate out dataset effects and not the primary phenotype of interest, as shown in

142 the CRC-WGS pooled dataset (Figure 1) and the other two datasets (Figure S1), suggesting that

143 the phenotype signal is weak in the presence of heterogeneity. 


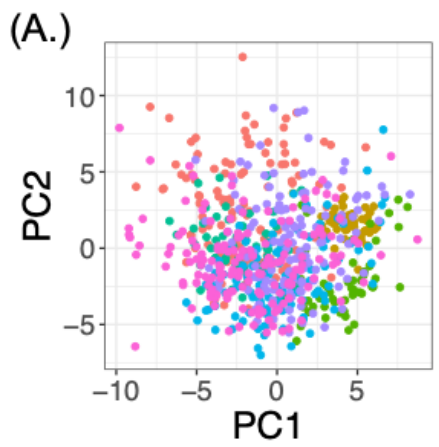

\section{Study}

Feng et al., 2015

Hannigan et al., 2015

Thomas et al., 2018a

Thomas et al., 2018b

Vogtmann etal., 2016

Yu et al., 2015

Zeller et al., 2014
(B.)

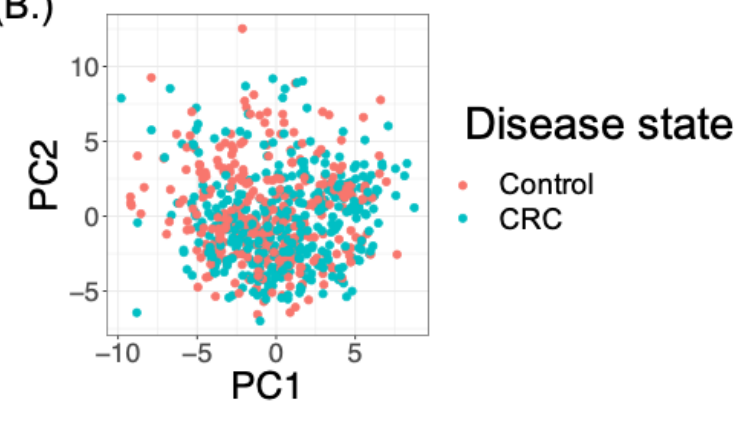

144

145 Figure 1. First two principal components from the CRC-WGS study. PCA was applied to OTU data from the CRC-WGS dataset, and samples were plotted along the first 2 PCs with colors in (A) indicating dataset membership and colors in (B) representing colorectal cancer More generally, the top 10 PCs from OTUs (Figure 2A and Figure S2) were highly correlated with technical variables, e.g., dataset label has a Pearson correlation of 0.87 with PC2 in CRC-16S and 0.45 with PC2 in CRC-WGS, and, to a lesser extent, biological variables unrelated to the phenotype of interest, e.g., sex (CRC-WGS: $\rho=0.10$ with PC5; CRC-16S: $\rho=$ 0.11 with PC5) and age (AGP: $\rho=0.16$ with PC2; CRC-WGS: $\rho=0.14$ with PC2; CRC-16S: $\rho=$ 0.16 with PC1). Other noteworthy correlations among the top 10 PCs include country of the host (CRC-WGS: $\rho=0.47$ with PC2), DNA extraction kit (CRC-WGS: $\rho=0.42$ with PC2), collection year (AGP: $\rho=0.48$ with PC5), and sequencing instrument (AGP: $\rho=0.45$ with PC5; CRC-16S: $\rho=0.56$ with PC1). Unlike the many technical and biological covariates, the phenotypes of interests were poorly or moderately correlated with the top $10 \mathrm{PCs}$ and include antibiotic consumption in the AGP ( $\rho=0.12$ with PC3) and CRC status (CRC-WGS: $\rho=0.24$ with PC3; CRC-16S: $\rho=0.20$ with PC4), and BMI in the AGP $(\rho=0.52$ with PC5). interest were significantly correlated with technical and biological covariates, which means that 
164 these covariates could potentially confound the ability to predict phenotypes. For example, the

165 country of host, DNA extraction kit, and dataset were significantly correlated to colorectal

166 cancer status in CRC-WGS ( $\rho=0.26 ; \rho=0.10$, and $\rho=0.13$, respectively) and sequencing

167 instrument and collection year in the AGP were significantly correlated with BMI $(\rho=0.45$ and $\rho$

$168=0.76$, respectively)

169 Compared to no transformation, CLR transformation resulted in more normally

170 distributed data (Figure S3), and stronger correlations between each of the top 10 PCs and

171 phenotypes of interest and technical covariates (Figure 2B, 2D and S2). For example, without

172 CLR transformation, antibiotic consumption in AGP was significantly correlated with the first 6

173 PCs that collectively explain 33\% of microbiome variance (p-value $\leq 0.02$ for each PC

174 significantly correlated, where the first 10 PCs were tested for correlations). However after CLR,

175 antibiotic consumption was significantly correlated with all the first 10 PCs that collectively

176 explain $50 \%$ of microbiome variance ( $\mathrm{p} \leq 0.01$ for each of the 10 PCs tested). Similarly, CRC

177 status in CRC-WGS was initially significantly correlated with PCs 1,3 and 9 ( $\mathrm{p} \leq 0.03$ for each

178 PC) that collectively explain $17 \%$ of microbiome variance, but after CLR, CRC status was

179 significantly correlated with PCs 1 through 5 ( $\mathrm{p} \leq 0.01$ for each PC) that collectively explain

$18031 \%$ of microbiome variance. Similar trends were found with BMI in AGP (Figure 2B) but not

181 with CRC in CRC-16S (Figure S2), although correlations significantly increase in magnitude for

182 all three datasets across all covariates Figure S4). Technical variables were more correlated with

183 top PCs than phenotypes of interest after CLR: the strongest correlations in CLR-transformed

184 AGP, CRC-WGS, and CRC-16S pooled datasets were between the first two PCs (which explain

$18514 \%, 12 \%, 15 \%$ of microbiome variance, respectively) and the dataset label (CRC-WGS: $\rho=$ 


\section{No transformation}

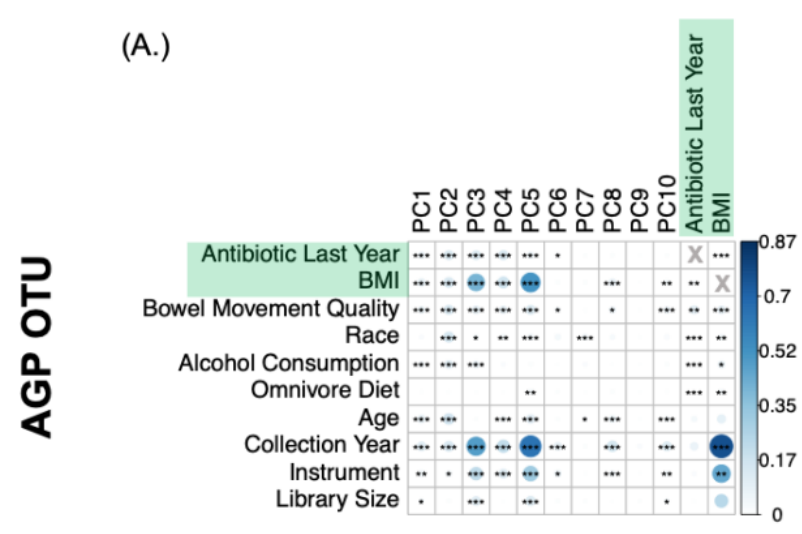

(C.)

\section{CLR transformation}

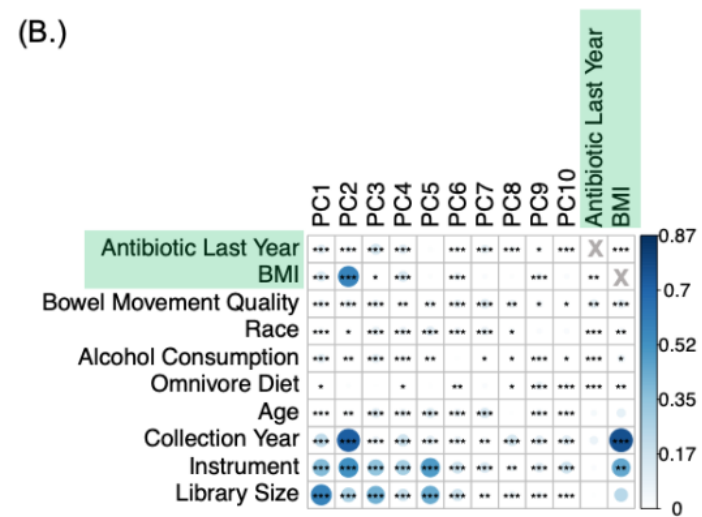

(D.)

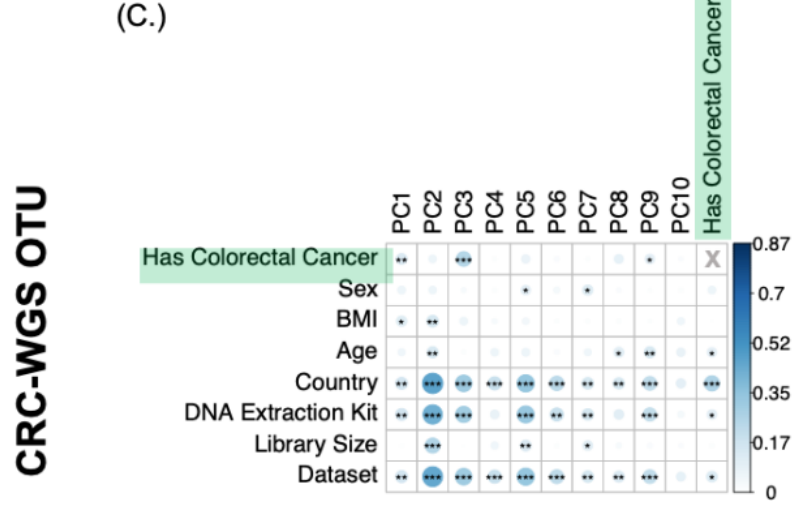

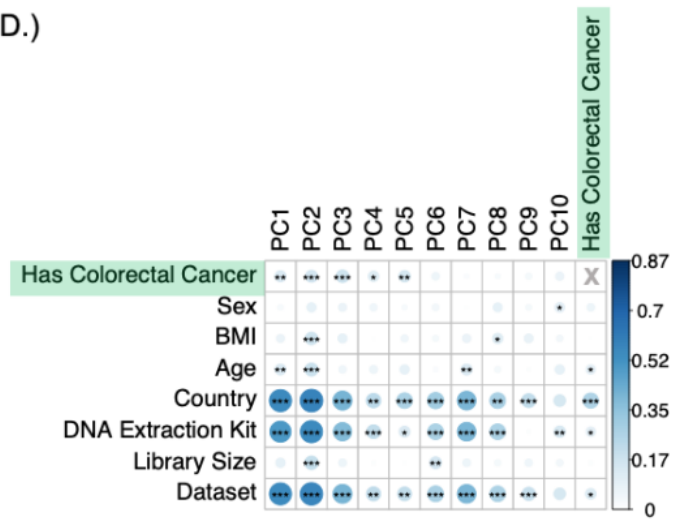

Figure 2._Microbiome data is affected by technical and biological variables. The first 10 PCs are correlated with variables measured in each of the studies, including dataset label, library size, DNA extraction kit used, country of origin, age, body mass index (BMI), sex, and colorectal cancer status (CRC). CLR reveals stronger correlations between the top principal components and confounding covariates compared to no transformation. The size and color of the circles in each cell indicates the magnitude of correlation and black asterisks indicate the significance of the Pearson correlation of the PCs with each of the variables. The color bar at right of each plot represents the range of correlations observed across all datasets. [*,**,*** indicate $p$-values as 
199 follows: $10^{-2}<p<0.05,10^{-3}<p<10^{-2}, 10^{-4}<p<10^{-3}, p<10^{-4}$ ]. See Figures S2 and S5 for

200 similar analyses for the CRC-16S joined dataset and 7-mers.

We also assessed the impact of $k$-merization on the correlation of variables with top PCs.

203 Similar to OTUs, CLR transformation of $k$-mers results in more significant correlations

204 compared to no transformation. In fact, the correlations of technical variables with PCs were

205 stronger for $k$-mers than OTUs for all three datasets (Figure S5). For example, sequencing

206 method is significantly correlated with CRC-16S PCs that collectively explained $21 \%$ of

207 variance in OTUs, but $49 \%$ of variance in $k$-mers. PCs significantly correlated with CRC status

208 in CRC-16S, by contrast, only collectively explained 16 and $18 \%$ of variance in OTUs and $k$ -

209 mers, respectively.

210

211 Background noise correction increases variance explained by phenotype while reducing

212 variance explained by confounders

213 We assessed how successfully background noise correction methods can remove

214 variation due to technical and biological confounders without sacrificing the phenotype of

215 interest, as this represents the net benefit of applying background noise correction (Figure 3A

216 and 3B, Figure S6). We focused on $k$-mers for this analysis because they show the most

217 sensitivity to technical confounders. The supervised background noise correction methods we

218 applied were $\mathrm{BMC}^{49}, \mathrm{ComBat}^{51}, \operatorname{limma}^{50}$. When testing $\mathrm{BMC}^{49}, \mathrm{ComBat}^{51}$ and limma ${ }^{50}$, we

219 correct for the primary contributor of heterogeneity in each dataset: sequencing instrument and

220 source study in the AGP and CRC datasets respectively, since these variables were the most

221 correlated with the top PCs (Figure 2).

Additionally, we assessed the ability of two variants of unsupervised correction

223 approaches in which PCA correction is applied after CLR: one in which data is corrected for the 
224 number of PCs that maximizes phenotype prediction accuracy and another in which data is

225 corrected for a fixed and arbitrary number of PCs. We refer to these two variants as tuned-PCA

226 and fixed-PCA, respectively (see Methods). Tuned-PCA uses a validation set to determine the

227 optimal number of PCs that maximize prediction accuracy while fixed-PCA correction corrects

228 for the first three PCs. The choice of three PCs for this analysis was arbitrarily selected to avoid

229 completely throwing away the signal associated with the phenotype of interest. We analyzed the

230 effect of higher numbers of PCs in the supplement (Figure S7). We included the fixed-PCA

231 approach in order to evaluate the ability of a correction method agnostic to the phenotype signal.

232 Finally, we also applied a supervised correction in which the primary contributors of

233 heterogeneity were regressed out, an approach we term in this paper as Direct Covariate

234 Correction (DCC) (see Methods). Because it is the only method that explicitly adjusts for the

235 primary potential confounders, DCC serves as a baseline assessing fixed-PCA correction, which

236 may not fully correct these primary confounders but on the other hand may correct for additional

237 confounders.

The proportion of variance explained by phenotype after applying each method is

239 quantified by modeling the $k$-mers as an outcome in a linear mixed model that jointly considers

240 all measured variables both biological and technical, and then estimating the fraction of variance

241 explained by each of these variables (see Methods). Tuned and fixed PCA correction resulted in

242 the greatest increase in proportion of variance explained by phenotypes of interest in all datasets

243 relative to no correction or DCC, respectively, and also relative to all other methods (Figure 3

244 and Figure S6). By contrast, $\operatorname{limma}^{50}$ and $\mathrm{BMC}^{49}$, produced a minimal, albeit significant,

245 increase in the proportion of variance explained by the phenotypes of interest in AGP and

246 insignificant increase or decrease in variance explained by CRC in CRC-WGS and CRC-16S. In 
247 the case of CRC-WGS and CRC-16S, ComBat ${ }^{51}$ significantly reduces the variance that can be 248 explained by CRC compared to uncorrected data $(\mathrm{p}<0.05)$ (Figure 3C and Figure S6).

Additionally, we computed the ratio of variance explained by the phenotype of interest

250 versus variance explained by the confounders (i.e., sequencing instrument, collection year, race,

251 diet, alcohol consumption, type of bowel movement, library size, and age). Compared to

252 uncorrected data, the tuned-PCA correction approach resulted in an increase in mean ratio by a

253 factor of 6.9 and 1.2 (Figures 3B, 3D, and S6) for the AGP and CRC-WGS datasets,

254 respectively, but resulted in a reduction for CRC-16S by a factor of 0.74 . Fixed-PCA performed

255 better than DCC in AGP by a factor of 3.5, but performed worse than DCC in CRC-WGS and

256 CRC-16S by factors of 0.49 and 0.44 , respectively (Figures 3B, 3D, and S6). In AGP and CRC-

257 WGS, ComBat ${ }^{51}$, limma ${ }^{50}$, and $\mathrm{BMC}^{49}$ also had significantly increased ratios, but tuned PCA

258 performed the best. In CRC-16S, only ComBat ${ }^{51}$ resulted in an increased ratio compared to

259 uncorrected data (increased by a factor of 1.3) (Figure S6).

260 We conclude from these findings that existing background noise correction methods

261 addressed variance due to confounders at the cost of the signal relevant to the phenotype of

262 interest. Both the fixed and tuned-PCA correction were able to increase the variance attributed to

263 phenotypes of interest across all datasets for the majority of features. 


\section{AGP 7-mers}

(A.) Variance explained by $\mathrm{BMI}$ and antibiotic consumption

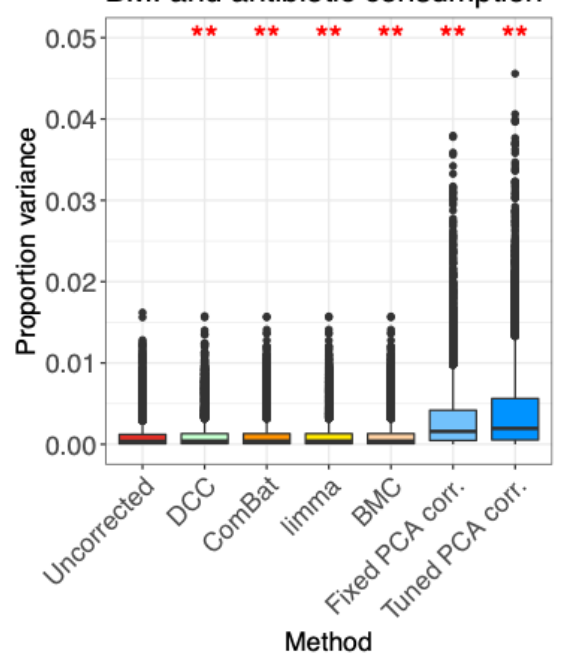

(B.)

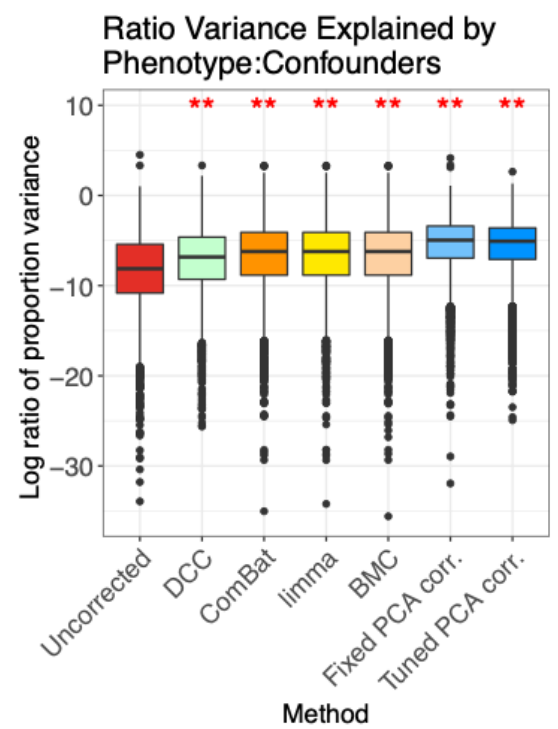

\section{CRC-WGS 7-mers}

(C.) Variance explained by colorectal cancer status

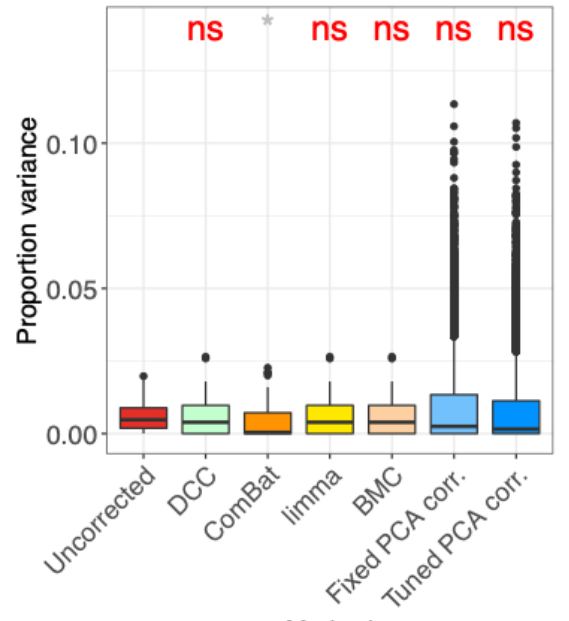

Method
(D.)

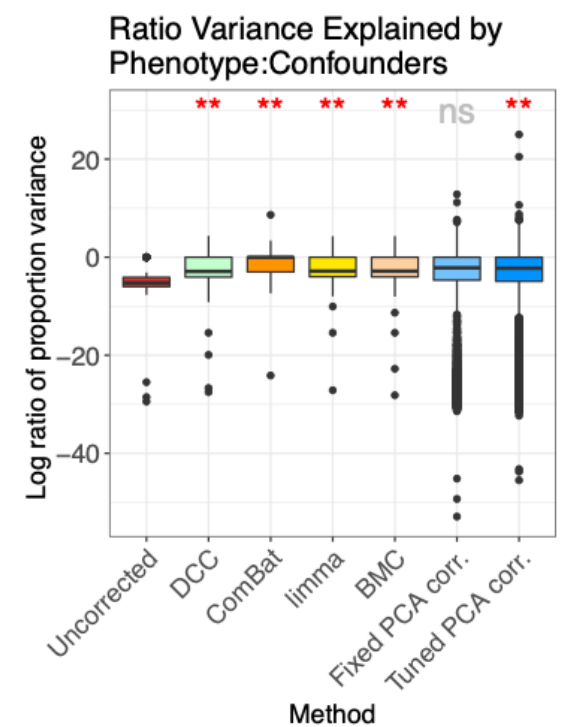

271 Figure 3. Proportion of variance explained by phenotypes of interest versus confounders.

272 We plotted the distribution of the proportion of variance in each of the 7-mer features that is

273 explained by (a) antibiotic consumption and BMI in the AGP cohort, and by (c) colorectal cancer

274 status in the CRC-WGS joined datasets. We plotted the $\log 2$ ratio of that same proportion by the

275 proportion explained by confounders, including DNA extraction kit, sequencing instrument, and

276 sex in the (b) AGP cohort and (d) the CRC-WGS joined datasets. Each point represents one

277 feature in the 7-mer matrix table. [ns, ${ }^{*}, * *, * * *$ indicate $p$-values as follows: non-significant at 
0.05 threshold, $10^{-2}<p<0.05,10^{-3}<p<10^{-2}, 10^{-4}<p<10^{-3}, p<10^{-4}$, respectively, in a

279 Wilcoxon rank sum test comparing each method to the baseline]. All methods' baseline was

280 Uncorrected except fixed-PCA, which was compared to DCC as a baseline. Refer to Figure S6

281 for the equivalent analysis for WGS-16S.

Impact of data transformations and background noise correction on phenotype prediction

We evaluated the impact of each correction approach on prediction of a host's colorectal

cancer status, antibiotic consumption, and BMI when using either OTUs or $k$-mers as input

hypothesized that, in scenarios when phenotype is not directly correlated to other covariates,

cases where covariates confound the phenotype directly, these approaches may not improve, and

even reduce, phenotype prediction accuracy because correction for confounders would also result

291 in removal of the phenotype of interest.

Among OTUs, all methods ${ }^{49-51}$ either had no effect or significantly reduced accuracy for

293 all phenotypes, compared to no correction (Figure 4). The reduction in accuracy is expected

294 when phenotypes of interest are confounded by covariates, as observed in previous sections.

295 Notably, fixed-PCA correction resulted in the greatest decrease in phenotype prediction

296 accuracy, even relative to its comparable baseline, DCC. Figure S7 shows that the AUC for

297 phenotype prediction increases monotonically as the first three PCs are regressed out. However,

298 if too many PCs are regressed out, then, phenotype prediction accuracy starts to decrease

299 indicating PCs associated with the phenotypic signal were removed.

301 BMI in AGP and CRC status in CRC-WGS, but in the case of antibiotic history in AGP and

302 CRC in CRC-16S, they had no effect or significantly reduced accuracy compared to no 
304 showed the greatest improvement in phenotype prediction for most datasets and phenotypes

305 (Figure 4).

Although $k$-mers showed lower overall phenotype prediction accuracy compared to

307 OTUs (Figure 4), PCA correction was able to make $k$-mers into a feasible predictive input

308 feature that has comparable prediction accuracy to OTUs. Thus, in scenarios where technical

309 covariates account for more microbiome variation than the phenotype of interest does, as

310 observed with $k$-mers, using a background noise correction strategy can significantly improve

311 phenotype prediction accuracy.

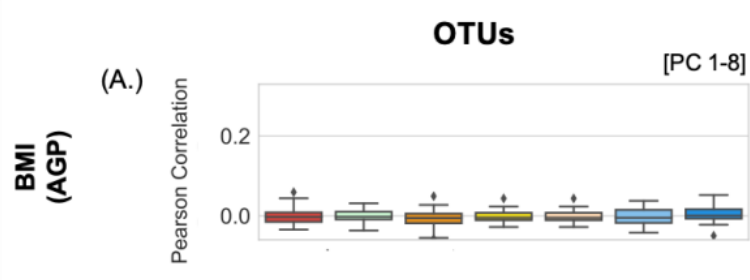

(C.)
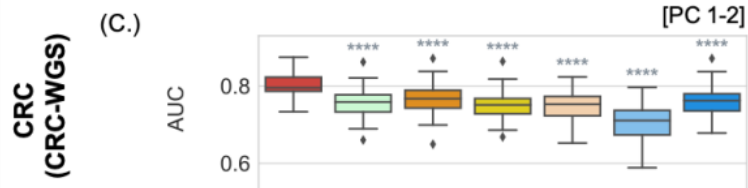

(E.)

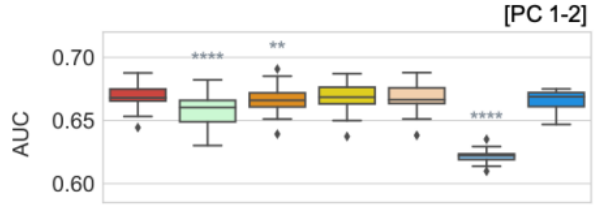

造

(G.)

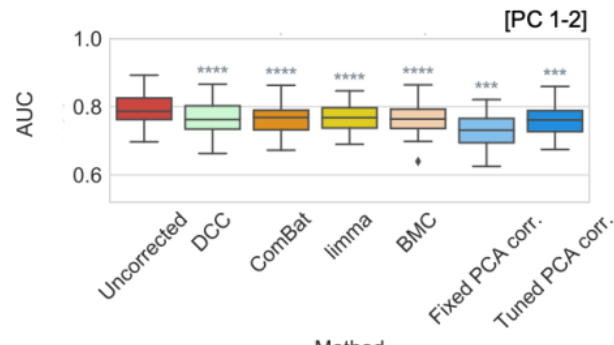

312

\section{3}

314

315
(B.)

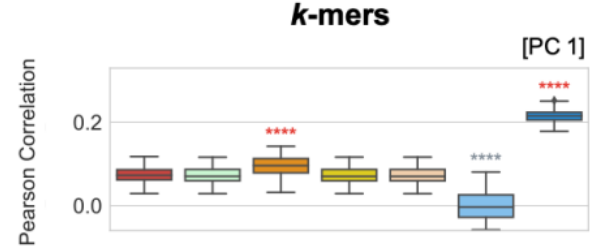

(D.)

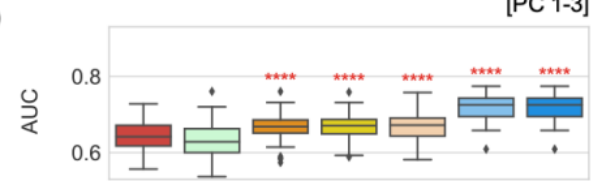

(F.)

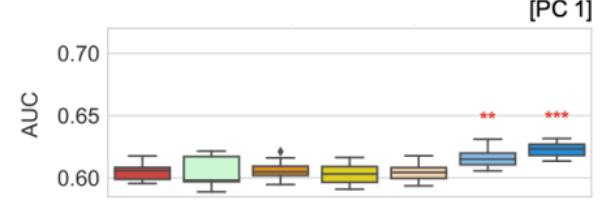

(H.)

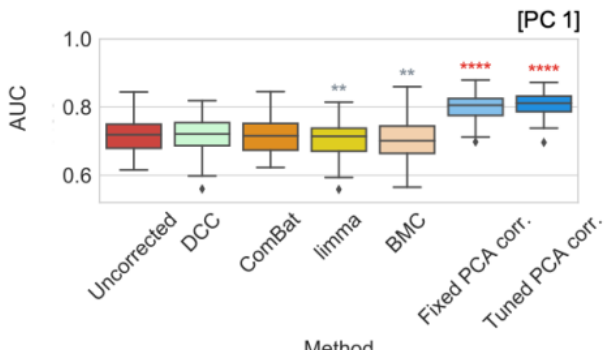

Figure 4. Impact of confounder correction approaches on phenotype prediction accuracy.

The distribution of AUC or Pearson correlation in a cross-validated prediction model using either uncorrected data or data after applying one of the following covariate correction approaches: 
316 DCC, ComBat ${ }^{51}, \operatorname{limma}^{50}, \mathrm{BMC}^{49}$, and fixed-PCA correction with three PCs regressed out, and

317 tuned-PCA correction where the number of PCs regressed out is a tuned hyperparameter.

318 Distributions with a significantly higher mean compared to the uncorrected data are indicated

319 with red asterisks, and distributions with a significantly lower mean compared to the uncorrected

320 data are indicated with grey asterisks. The number of asterisks corresponds to the significance of

321 a paired t-test between the uncorrected and corrected data (Methods). [ns, ${ }^{*}, * *, * * *$ indicate $p$ -

322 values as follows: non-significant at 0.05 threshold, $10^{-2}<p<0.05,10^{-3}<p<10^{-2}, 10^{-4}<p<10^{-}$

$323{ }^{3}, p<10^{-4}$, respectively, in a Wilcoxon rank sum test comparing each method to the baseline].

324 All methods' baseline was Uncorrected except Fixed-PCA, which was compared to DCC as a

325 baseline. Shown at top right is the number of PCs regressed out in the Tuned-PCA method

Discussion

328 The ability to predict human phenotypes from metagenomic data is important to establish

329 biomarkers of disease and the subsequent development of therapeutics. However, a major issue

330 that impacts accurate prediction is the presence of confounders and systemic background noise

331 both within ${ }^{26}$ and across studies ${ }^{15,31}$. In this paper, we investigated the ability of existing

332 approaches to correct for sources of background noise in microbiome data and evaluated the

333 potential utility of an unsupervised approach - PCA correction on CLR-transformed data.

PCA correction has been effective in correcting for unwanted variation in human genetic

335 data and morphological data ${ }^{65-68}$, but to date has not been applied to correct for such noise in

336 microbiome data. Yet, we and others have found that top principal components in multiple

337 datasets are correlated with numerous potential sources of unwanted noise such as host

338 genetics $^{69}$, ethnicity of the host ${ }^{70}$, and also abiotic factors like temperature ${ }^{71}$, suggesting that PCA

339 correction may be an effective unsupervised correction approach. We found that regressing out

340 the top PCs after applying a CLR transformation can address multiple issues simultaneously:

341 first, this approach can reduce variance associated with technical and biological factors while 
342 improving the proportion of variance attributable to the phenotype of interest and resulting in

343 improved ability to predict phenotypes (Figure 4). Additionally, the output from PCA correction

344 can further be used for downstream analyses such as differential abundance analysis. We found

345 that the extent of background noise differs from one dataset to another, and thus the ideal number

346 of PCs to regress out with either a fixed or tuned PCA correction varies for each dataset. For

347 example, the top five PCs in the AGP dataset are significantly correlated with technical

348 variables, whereas the top nine PCs in the CRC-WGS are correlated with technical variables.

The application of CLR transformation can address many attributes of microbiome data

350 that make it difficult to model including sparsity, non-normality (Figure S3) and dependency of

351 features, which existing unsupervised approaches designed for non-microbiome data ${ }^{43-45}$ are ill-

352 equipped to deal with. The application of CLR to microbiome data has been broadly

353 recommended ${ }^{53,72}$ and is part of a suite of methods known as Compositional Data Analysis

354 (CoDA $)^{73,74}$ to address the dependency between features inherent to compositional data.

355 However, the adoption of CLR in the microbiome field has not been uniform. Recently,

356 McLaren at al. ${ }^{15}$ argued that CoDA methods can make microbiome data invariant to bias and

357 suggests that it is underutilized within the field. Specifically, McLaren et al. ${ }^{15}$ found that that

358 ratio-based analyses could remove intra-study bias, though did not address its effect on multiple

359 datasets that are pooled together or large datasets with heterogeneous sampling procedures such

360 as the AGP. Here, we provide the first systematic investigation into the effect of how CLR in

361 combination with PCA can remove inter-study and intra-study bias. We hypothesized that

362 applying CLR transformation will more readily reveal the covariates that introduce background

363 noise across and within heterogeneous datasets because these contributors of bias (e.g. DNA

364 extraction method, sequencing instrument, etc.) have a multiplicative effect on relative 
365 abundances ${ }^{15}$. We found that indeed relationships between the microbiome and such variables is

366 more apparent after CLR transformation (Figure 2), which is consistent with the multiplicative

367 nature of bias expounded in McLaren et al. ${ }^{15}$ because the multiplicative bias becomes additive in

$368 \log$ space, such that PCA is able to capture the bias in the top PCs as a shift in the centroid of

369 samples plotted for a given dataset.

370 The supervised approaches ${ }^{49-51}$ are beneficial in that they directly remove confounding,

371 potentially at the cost of phenotype prediction, while unsupervised approaches are beneficial in

372 correcting for both measured and unmeasured factors of microbiome. Correcting for confounders

373 naturally tends to result in removal of phenotype signal, as is the case in ComBat ${ }^{51}$ and fixed-

374 PCA, which may be more effectively removing confounding effects at the cost of reduced

375 proportion of variance explained by phenotype (Figure 3). Tuned-PCA protects the phenotype

376 effect by removing up to the first PC that would significantly remove phenotype signal.

377 However, caution must be taken when using tuned-PCA in the presence of strong confounding as

378 it may not remove all confounding in order to protect the phenotype effect. In these scenarios,

379 one should consider either a liberal correction of confounding by correcting for more PCs or

380 subsampling the data such that cases and controls are matched for known confounders as is done

381 in Vujkovic-Cvijin et al. ${ }^{26}$.

382 In addition to optimizing the number of PCs for regression, we similarly identified the

383 best Random Forest parameters and data transformations for the ComBat ${ }^{51}$, limma ${ }^{50}$, and $\mathrm{BMC}^{49}$

384 methods to fully evaluate the strengths of each approach (e.g. log transformed input was optimal

385 for $\mathrm{ComBat}^{51}$ ). Note that we did not compare methods to percentile normalization ${ }^{36}$, which is a

386 supervised approach recently proposed for robust biomarker discovery rather than phenotype

387 prediction. Percentile normalization requires using all the control samples in one dataset to 
determine the percentiles for all the case samples in that same dataset, and so it is not

immediately obvious how to apply this to a phenotype prediction model in which held out data is exciting area of methodological development.

We compared the prediction ability and impact of background noise correction methods on OTUs versus $k$-mers. $K$-mers are a convenient reference-free alternative to taxonomic features in that they produce non-sparse features that are also normally distributed at small values of $k$.

Previous work has found that $k$-mers of sizes six and seven from microbiome data are predictive of phenotypes ${ }^{75}$. However, $k$-mers are limited because they are usually not directly interpretable

397 biological features, although they can still be used as biomarkers of disease. This limitation may

398 be a reason why OTUs outperform $k$-mers in phenotype prediction accuracy (Figure 4). It is

399 crucial to note however, that $k$-mers may provide a better signature of technical artifacts like

400 PCR bias $^{76,77}$ and are also known to be protocol specific ${ }^{78}$. Thus, this may explain why for both

401 16S and WGS data, $k$-mers had higher correlations with technical variables compared to OTUs

402 (Figures 2, S2 and S5). This aspect of $k$-mers offers justification for why PCA correction is

403 particularly effective with $k$-mers.

As we hypothesized, in scenarios where the phenotype of interest is not directly

405 correlated with other covariates, supervised approaches for batch correction and PCA correction 406 yield better prediction than uncorrected data. This was true for $k$-mers where non-phenotypic

407 variables were not as correlated with the phenotypes of interest compared to OTUs. This 408 observation offers another justification for why PCA correction improved phenotype prediction 409 in $k$-mers whereas in OTUs all correction methods reduced phenotype prediction. The difference 410 in distribution of cases and controls between different studies is more drastic for some pooled 
411 datasets than others, reflecting a higher possibility of confounding (Table S1). We note that even

412 though application of background noise correction approaches to OTUs does not always improve

413 phenotype prediction accuracy, by applying correction, the true causal OTUs for the phenotype

414 may be easier to identify with fewer false positives $^{31}$.

415 Background noise correction is becoming increasingly important as the microbiome field

416 matures and new datasets become available. One exciting future application of correction that we

417 foresee is in microbiome wide association studies in which microbiome genomic polymorphisms

418 are associated with human phenotypes ${ }^{79,80}$. Such a scenario may benefit from background noise

419 correction since population structure may play a considerable confounding role ${ }^{81}$. As researchers

420 consider the best approach for background noise correction for their specific research questions,

421 they must weigh the tradeoffs between addressing confounding while also maintaining as much

422 of the phenotype signal as possible. There is no single solution that will address all problems, but

423 at minimum researchers should perform careful forensics to investigate the nature and

424 pervasiveness of confounders in their data. In this manner, consistent and robust inferences can

425 be made across multiple studies, moving us towards the goal of accurate phenotype prediction

426 from microbiome data. 
434 Methods

435 Datasets

436 Raw 16S fastq files were downloaded from the NCBI Sequence Read Archive (SRA)

437 with study accessions PRJEB11419 for the American Gut Project, and PRJNA290926 (Baxter et

438 al. ${ }^{58}$ ) and PRJEB6070 (Zeller et al. ${ }^{82}$ ) for CRC-16S. Fastq files for Zackular et al. ${ }^{60}$ from CRC-

$43916 \mathrm{~S}$ were obtained from http://mothur.org/MicrobiomeBiomarkerCRC/. The raw WGS fastq files

440 for CRC-WGS were downloaded from SRA with study accessions PRJEB12449 (Vogtmann et

441 al. $^{62}$ ), PRJEB10878 (Yu et al. ${ }^{63}$ ), PRJEB7774 (Feng et al. ${ }^{61}$ ), PRJNA447983 (Thomas et al.

442 Italian validation cohorts ${ }^{1}$ ), PRJEB6070 (Zeller et al. ${ }^{82}$ ), and PRJNA389927 (Hannigan et al. ${ }^{64}$ ).

443 Processed OTU data for the AGP was obtained from Qiita study id 10317 (EBI submission

444 ERP012803). OTU profiles from CRC-16S were obtained from the MicrobiomeHD database

445 (Duvallet et al. $^{8}$ ). Taxonomic profiles for CRC-WGS were obtained through the R package

446 curatedMetagenomicData ${ }^{83}$ which used MetaPhlAn2 ${ }^{84}$.

\section{Raw Sequence Processing}

Features in metagenomic data can be defined in two broad ways, both high-dimensional:

450 reference-based approaches and reference-free approaches. Reference-based approaches cluster

451 sequenced reads based on a defined threshold and assign taxonomy by aligning reads to

452 reference genomes. Reference-free approaches, sort reads into bins that are defined

453 independently of known genomes, i.e. $k$-mers, short strings of length $k$ that can be obtained

454 directly from read sequences, which are increasingly popular in microbiome data analyses and

455 have been used by several studies to do prediction ${ }^{85-88} . K$-mers offer a powerful alternative

456 approach to more commonly used Operational Taxonomic Units (OTUs) because they do not

457 rely on a reference database of genomes and do not require identifying a set of parameters to

458 determine OTUs ${ }^{89}$.

460 To compute $k$-mer abundances, raw sequences were input into the $k$-mer counting algorithm

461 Jellyfish $2.3 .0^{90}$ with default parameters except for a hash of 10 million elements and canonical

$462 k$-mers with size of 5,6,7 or 8. Prior work has shown that $k$-mer sizes of 6 and 7 are predictive of

463 phenotype ${ }^{56}$. The resulting $k$-mer abundance table is then converted to a composition such that 
each sample sums to 1 to account for different reads depths across samples. Taxonomic profiles were similarly converted to compositions.

\section{Centered log ratio transformation}

The centered log ratio (CLR) transformation is a compositional data transformation that takes the $\log$ ratio of between observed frequencies and their geometric means. This is done within each sample where relative frequencies of different taxa are measured and sum to 1 . This can be written in mathematical form as:

$$
\begin{gathered}
\operatorname{clr}(x)=\left[\log \frac{\mathrm{x}_{1}}{\mathrm{G}(\mathrm{x})}, \log \frac{\mathrm{x}_{2}}{\mathrm{G}(\mathrm{x})}, \ldots, \log \frac{\mathrm{x}_{\mathrm{n}}}{\mathrm{G}(\mathrm{x})}\right] \\
=\left[\log x_{1}-\log G(x), \log x_{2}-\log G(x), \ldots, \log x_{n}-\log G(x)\right] \\
\mathrm{G}(x)=\left(\prod_{i=1}^{N} x_{i}\right)^{1 / N}
\end{gathered}
$$

Here, $\boldsymbol{x}$ is a vector representing the abundance of microbiome features in a single sample, and $G(x)$ represents the geometric mean. Prior to applying PCA, the CLR-transformed values are standardized such that all samples have a mean of 0 and variance of 1 . The Gaussian-like distribution of CLR-transformed microbiome compositional data is shown in Figure $\mathbf{S 3}$.

\section{Background noise correction methods}

The existing supervised approaches for background noise correction compared in this study include batch mean centering (BMC) $)^{49}, \mathrm{ComBat}^{51}$, and limma $^{50}$ applied to relative abundance data. ComBat ${ }^{51}$ assumes data is cleaned and normalized prior to batch effect removal. It's common to add a pseudocount of 1 to 0 observations so that one can apply a log transform in the normalization prior to ComBat ${ }^{51}$ (as described in Gibbons et al. ${ }^{31}$ ). We followed this same procedure with both OTU and $k$-mer, and applied ComBat ${ }^{51}$ to the log of relative abundance data. For limma, batch mean centering (BMC), and Direct Covariate Correction (DCC) we used the relative abundance. The CLR transformation and PCA-Correction used the relative abundance of $k$-mers and taxonomic features. The equation used to regress out confounding covariates in DCC is as follows: 
492 Where the original feature matrix $X$ with $m$ features and $n$ samples is the outcome of a linear

493 model with covariate associated coefficient matrix $\beta$, dummy matrix $C$ with each row

494 representing one of the $b$ possible values of the confounding covariate, and $\epsilon$, the residual

495 matrix. The residual matrix $\epsilon$ is the covariate-corrected feature matrix.

496 In PCA correction, top PCs computed from the CLR transformed $k$-mer or OTU relative

497 abundance tables are regressed out. The CLR transformation cancels out the multiplicative bias ${ }^{15}$

498 within each study by taking a ratio of features to the geometric mean of features that are all

499 impacted by the same study-specific multiplicative bias. The transformation accentuates the

500 difference in bias across studies by smoothing out the intra-study bias, thereby allowing PC

501 regression to account for the confounding across studies. In the fixed-PCA correction, a set

502 number of PCs are regressed out from the microbiome data. In the main figures we show results

503 after regressing up to three PCs. We show results regressing up to 9 PCs in the supplement.

504 Alternatively, the tuned-PCA correction uses a train-validation-test approach to tune two

505 hyperparameters: the optimal number of PCs to regress out $p$, and, when using $k$-mers, the

506 optimal $k$. The same portion of data used for validation in the Random Forest tuning is used for

507 tuning the PCA correction hyperparameters, where the tuned Random Forest hyperparameters

508 are fixed before tuning $p$ and $k$. To determine the number of PCs that optimize phenotype

509 prediction, PCs 1 through $p$ were regressed out of the input data with $p$ ranging from 1 to 20 . The

$510 \quad p$ that produces the highest AUC or Pearson correlation in phenotype prediction (method of

511 prediction model described below) in validation was selected. The same procedure is done with $k$

512 where values between 5 and 8 are tested (only $k$-mer sizes 6 and 7 were tested for CRC-WGS)

513 The reported performance is based on the remaining $20 \%$ set aside for testing.

\section{Variance components analysis}

516 We performed variance components analysis before and after correction to show how batch

517 correction impacts biological signal of interest and technical signal. Specifically, a linear mixed

518 model was fit to the data where the outcome was abundance assigned to a taxa or $k$-mer, and the

519 variables of the model were various biological and technical variables obtained from a study's

520 metadata. The outcome was represented by a vector (with dimensions $t$ features $\mathrm{x} 1$ ), and the

521 biological and technical covariates were represented by a design matrix (with dimension of

522 number of covariates $c$ x number of samples $n$ ). Continuous variables, e.g. BMI, and categorical 
523 variables with two or less levels (e.g. sex) were modeled as fixed effects while categorical

524 variable with more than two levels (e.g. ethnicity) were modelled as random effects. This LMM

525 model was computed with the R package variancePartition ${ }^{91}$. For example, in the AGP, the

526 following LMM was implemented:

Feature $\sim$ BMI + age + library size $+(1 \mid$ antibiotic consumption $)+(1 \mid$ alcohol consumption $)+(1 \mid$

collection year $)+(1 \mid$ instrument $)+(1 \mid$ bowel movement quality $)+(1 \mid$ diet $)+(1 \mid$ sex $)+(1 \mid$ race $)$

For AGP, sequencing instrument, collection year, race, alcohol consumption, diet, bowel movement and antibiotic consumption were modeled as random effects while library size, age, and BMI were modeled as fixed effects. For CRC-WGS, colorectal cancer status, dataset label, library layout, sequencing instrument, sequencing center, and DNA extraction kit, and sex were modeled as random effects and library size was modeled as a fixed effect. For 16S, dataset label, sequencing method, race, CRC status, and sex were modeled as random effects while library size and age were modeled as fixed effects.

\section{Phenotype prediction}

540 In CRC-16S and CRC-WGS, we predicted whether a sample comes from a host with colorectal

541 cancer or a healthy host. For the American Gut Project, we predicted whether a sample comes

542 from a host who took antibiotics in the previous year or a host who has not taken antibiotics in

543 the previous year. We also use the American Gut Project to predict body mass index (BMI).

545 We performed prediction of binary traits using Random Forest implemented in Scikit-learn ${ }^{92}$,

546 which has been previously employed successfully for predicting binary outcomes from

547 microbiome data $32,34,93,94$. We tuned four hyper-parameters of the Random Forest model in a grid

548 search using a train-validation-test strategy with $56 \%$ of samples in the meta-cohort used for

549 training, 24\% for validation of model hyper-parameters, and 20\% reserved for testing. These four

550 hyperparameters included the number of estimator trees (100,1000, or 1500), criterion (entropy

551 or gini), minimum samples per leaf $(1,5$, or 10$)$, and features $(0.1,0.3,0.5)$. Minimum samples

552 per split was set at 2 and max depth was set at 5, and default parameters otherwise. This was

553 performed in five-fold cross validation repeated ten-times to obtain confidence intervals on the 
554 area under the ROC curve (AUC), our metric of prediction accuracy. A similar train-validation-

555 test strategy was used for the linear regression model to select coefficients of the model where

556 accuracy was measured using Pearson correlation of the true BMI to the predicted BMI. The

557 difference in the distribution of prediction accuracy for both prediction tasks was quantified

558 statistically using a paired Student's t-test.

Code Availability

561 An implementation of PCA correction and phenotype classification is available at

562 https://github.com/garudlab/Microbiome_PCA_correction.

\section{Funding}

565 This research was supported in part by The National Science Foundation Graduate Research

566 Fellowship Program Award Number 1650604. The funders had no role in study design, data

567 collection and analysis, decision to publish, or preparation of the manuscript.

\section{Acknowledgements}

570 We thank members of the Halperin Lab and Garud Lab, as well as Michael R. McLaren for

571 helpful discussions.

572

\section{Author Contributions}

574 Conceived and designed the experiments: L.B., B.B., S.S., E.H., N.R.G. Performed the

575 experiments: L.B., B.B. Analyzed the data: L.B., B.B. Coded the pipeline: L.B. Contributed

576 analysis tools: E.H., N.R.G. Wrote the paper: L.B., B.B., S.S., E.H., N.R.G. All authors have

577 read and approved the manuscript. 


\section{References}

591 1. Thomas, A. M. et al. Metagenomic analysis of colorectal cancer datasets identifies cross-

592 cohort microbial diagnostic signatures and a link with choline degradation. Nat. Med. 25, $593 \quad 667-678(2019)$.

594 2. Ley, R. E. et al. Obesity alters gut microbial ecology. Proc. Natl. Acad. Sci. U. S. A. 102, $595 \quad 11070-11075(2005)$.

596 3. Ley, R. E., Turnbaugh, P. J., Klein, S. \& Gordon, J. I. Microbial ecology: Human gut 597 microbes associated with obesity. Nature 444, 1022-1023 (2006).

598 4. Jakobsson, H. E. et al. Short-Term Antibiotic Treatment Has Differing Long-Term 599 Impacts on the Human Throat and Gut Microbiome. PLoS One 5, e9836 (2010).

600 5. Jernberg, C., Löfmark, S., Edlund, C. \& Jansson, J. K. Long-term impacts of antibiotic 601 exposure on the human intestinal microbiota. Microbiology 156, 3216-3223 (2010).

602 6. Shaw, L. P. et al. Modelling microbiome recovery after antibiotics using a stability 603 landscape framework. ISME J. 13, 1845-1856 (2019).

604 7. Francino, M. P. Antibiotics and the human gut microbiome: Dysbioses and accumulation $605 \quad$ of resistances. Frontiers in Microbiology 6, 1543 (2016).

606 8. Duvallet, C., Gibbons, S. M., Gurry, T., Irizarry, R. A. \& Alm, E. J. Meta-analysis of gut 607 microbiome studies identifies disease-specific and shared responses. Nat. Commun. 8, $608 \quad$ (2017).

609 9. Qin, N. et al. Alterations of the human gut microbiome in liver cirrhosis. Nature 513, 59$610 \quad 64(2014)$.

611 10. D’Amore, R. et al. A comprehensive benchmarking study of protocols and sequencing 612 platforms for 16S rRNA community profiling. BMC Genomics 17, 55 (2016).

613 11. Hugerth, L. W. \& Andersson, A. F. Analysing microbial community composition through 614 amplicon sequencing: From sampling to hypothesis testing. Frontiers in Microbiology $\mathbf{8}$, $6151561(2017)$.

616 12. Pollock, J., Glendinning, L., Wisedchanwet, T. \& Watson, M. The madness of 617 microbiome: Attempting to find consensus 'best practice' for 16S microbiome studies. $618 \quad$ Applied and Environmental Microbiology 84, (2018).

619 13. Sinha, R. et al. Assessment of variation in microbial community amplicon sequencing by 
the Microbiome Quality Control (MBQC) project consortium. Nat. Biotechnol. 35, 10771086 (2017).

622 14. Sacristán-Soriano, O., Banaigs, B., Casamayor, E. O. \& Becerro, M. A. Exploring the links between natural products and bacterial assemblages in the sponge aplysina aerophoba. Appl. Environ. Microbiol. 77, 862-870 (2011).

15. McLaren, M. R., Willis, A. D. \& Callahan, B. J. Consistent and correctable bias in metagenomic sequencing experiments. Elife 8, (2019).

16. Brooks, J. P. et al. The truth about metagenomics: Quantifying and counteracting bias in 16S rRNA studies Ecological and evolutionary microbiology. BMC Microbiol. 15, (2015).

17. Zarrinpar, A., Chaix, A., Yooseph, S. \& Panda, S. Diet and feeding pattern affect the diurnal dynamics of the gut microbiome. Cell Metab. 20, 1006-1017 (2014).

18. Ma, J. et al. High-fat maternal diet during pregnancy persistently alters the offspring microbiome in a primate model. Nat. Commun. 5, (2014).

19. Rizzetto, L., Fava, F., Tuohy, K. M. \& Selmi, C. Connecting the immune system, systemic chronic inflammation and the gut microbiome: The role of sex. Journal of Autoimmunity 92, 12-34 (2018).

20. Amir, A. et al. Correcting for Microbial Blooms in Fecal Samples during RoomTemperature Shipping. mSystems 2, (2017).

21. Sipos, R. et al. Effect of primer mismatch, annealing temperature and PCR cycle number

641 22. Song, J. et al. Preservation Methods Differ in Fecal Microbiome Stability, Affecting 642 Suitability for Field Studies Downloaded from. 1, 21-37 (2016).

643 23. Kim, D. et al. Optimizing methods and dodging pitfalls in microbiome research.

644 Microbiome 5, (2017).

645 24. Costea, P. I. et al. Towards standards for human fecal sample processing in metagenomic 646 studies. Nat. Biotechnol. 35, 1069-1076 (2017).

647 25. Bartolomaeus, T. U. P. et al. Quantifying technical confounders in microbiome studies. 648 Cardiovasc. Res. (2020). doi:10.1093/cvr/cvaa128

649 26. Vujkovic-Cvijin, I. et al. Host variables confound gut microbiota studies of human 650 disease. Nature 587, 448-454 (2020). 
651 27. McDonald, D. et al. American Gut: an Open Platform for Citizen Science Microbiome Research. mSystems 3, (2018).

653 28. Wang, Y. \& Lê Cao, K.-A. Managing batch effects in microbiome data. Brief. Bioinform. $654 \quad 2019,1-17(2019)$.

655 29. Armour, C. R., Nayfach, S., Pollard, K. S. \& Sharpton, T. J. A Metagenomic Metaanalysis Reveals Functional Signatures of Health and Disease in the Human Gut Microbiome. mSystems 4, (2019).

30. Nayfach, S., Shi, Z. J., Seshadri, R., Pollard, K. S. \& Kyrpides, N. C. New insights from uncultivated genomes of the global human gut microbiome. Nature 568, 505-510 (2019).

31. Gibbons, S. M., Duvallet, C. \& Alm, E. J. Correcting for batch effects in case-control microbiome studies. PLoS Comput. Biol. 14, (2018).

32. Thomas, A. M. et al. Metagenomic analysis of colorectal cancer datasets identifies crosscohort microbial diagnostic signatures and a link with choline degradation. Nat. Med. 25, 667-678 (2019).

33. Su, X. et al. Multiple-Disease Detection and Classification across Cohorts via Microbiome Search. mSystems 5, (2020).

667 34. Pasolli, E., Truong, D. T., Malik, F., Waldron, L. \& Segata, N. Machine Learning Metaanalysis of Large Metagenomic Datasets: Tools and Biological Insights. PLOS Comput. Biol. 12, e1004977 (2016).

35. Asnicar, F. et al. Microbiome connections with host metabolism and habitual diet from 1,098 deeply phenotyped individuals. Nat. Med. (2021). doi:10.1038/s41591-020-01183-8

672 36. Gibbons, S. M., Duvallet, C. \& Alm, E. J. Correcting for batch effects in case-control microbiome studies. PLOS Comput. Biol. 14, e1006102 (2018).

674 37. Morgan, X. C. et al. Associations between host gene expression, the mucosal microbiome, and clinical outcome in the pelvic pouch of patients with inflammatory bowel disease. Genome Biol. 16, 67 (2015).

677 38. Pérez-Jaramillo, J. E. et al. Linking rhizosphere microbiome composition of wild and domesticated Phaseolus vulgaris to genotypic and root phenotypic traits. ISME J. 11, 2244-2257 (2017).

680 39. Kim, H. J., Li, H., Collins, J. J. \& Ingber, D. E. Contributions of microbiome and 
gut-on-a-chip. Proc. Natl. Acad. Sci. U. S. A. 113, E7-E15 (2016).

40. Nayfach, S. \& Pollard, K. S. Toward Accurate and Quantitative Comparative Metagenomics. Cell 166, 1103-1116 (2016).

41. Hiergeist, A. et al. Multicenter quality assessment of $16 \mathrm{~S}$ ribosomal DNA-sequencing for microbiome analyses reveals high inter-center variability. Int. J. Med. Microbiol. 306, 334-342 (2016).

42. Mallick, H. et al. Experimental design and quantitative analysis of microbial community multiomics. Genome Biology 18, 1-16 (2017).

43. Leek, J. T. \& Storey, J. D. Capturing heterogeneity in gene expression studies by surrogate variable analysis. PLoS Genet. 3, 1724-1735 (2007).

44. Gagnon-Bartsch, J. A., Jacob, L. \& Speed, T. P. Removing Unwanted Variation from High Dimensional Data with Negative Controls. (2013).

45. Rahmani, E. et al. Sparse PCA corrects for cell type heterogeneity in epigenome-wide association studies. Nat. Methods 13, 443-445 (2016).

46. Xu, L., Paterson, A. D., Turpin, W. \& Xu, W. Assessment and selection of competing models for zero-inflated microbiome data. PLoS One 10, e0129606 (2015).

47. Kaul, A., Mandal, S., Davidov, O. \& Peddada, S. D. Analysis of microbiome data in the presence of excess zeros. Front. Microbiol. 8, 2114 (2017).

49. Sims, A. H. et al. The removal of multiplicative, systematic bias allows integration of

50. Ritchie, M. E. et al. Limma powers differential expression analyses for RNA-sequencing and microarray studies. Nucleic Acids Res. 43, e47 (2015).

51. Johnson, W. E., Li, C. \& Rabinovic, A. Adjusting batch effects in microarray expression data using empirical Bayes methods. Biostatistics 8, 118-127 (2007).

709 52. Fernandes, A. D. et al. Unifying the analysis of high-throughput sequencing datasets: 
datasets are compositional: And this is not optional. Frontiers in Microbiology 8, (2017).

714 54. Martino, C. et al. A Novel Sparse Compositional Technique Reveals Microbial

715 Perturbations. mSystems 4, (2019).

716 55. Shi, P., Zhang, A. \& Li, H. Regression analysis for microbiome compositional data. Ann. Appl. Stat. 10, 1019-1040 (2016).

718 56. Lê Cao, K.-A. et al. MixMC: A Multivariate Statistical Framework to Gain Insight into 719 Microbial Communities. PLoS One 11, e0160169 (2016).

720 57. van den Boogaart, K. G. \& Tolosana-Delgado, R. Analyzing compositional data with R. Analyzing Compositional Data with R (Springer Berlin Heidelberg, 2013). doi:10.1007/978-3-642-36809-7

58. Baxter, N. T., Ruffin, M. T., Rogers, M. A. M. \& Schloss, P. D. Microbiota-based model improves the sensitivity of fecal immunochemical test for detecting colonic lesions. Genome Med. 8, 37 (2016).

59. Zeller, G. et al. Potential of fecal microbiota for early-stage detection of colorectal cancer. Mol. Syst. Biol. 10, 766 (2014).

728 60. Zackular, J. P., Rogers, M. A. M., Ruffin, M. T. \& Schloss, P. D. The human gut microbiome as a screening tool for colorectal cancer. Cancer Prev. Res. 7, 1112-1121

731 61. Feng, Q. et al. Gut microbiome development along the colorectal adenoma-carcinoma sequence. Nat. Commun. 6, 1-13 (2015).

62. Vogtmann, E. et al. Colorectal Cancer and the Human Gut Microbiome: Reproducibility with Whole-Genome Shotgun Sequencing. PLoS One 11, e0155362 (2016).

63. Yu, J. et al. Metagenomic analysis of faecal microbiome as a tool towards targeted noninvasive biomarkers for colorectal cancer. Gut 66, 70-78 (2017).

737 64. Hannigan, G. D., Duhaime, M. B., Ruffin, M. T., Koumpouras, C. C. \& Schloss, P. D.

740 65. Novembre, J. et al. Genes mirror geography within Europe. Nature 456, 98-101 (2008).

741 66. Price, A. L., Zaitlen, N. A., Reich, D. \& Patterson, N. New approaches to population stratification in genome-wide association studies. Nature Reviews Genetics 11, 459-463 743 (2010). 
744 67. Tucker, G., Price, A. L. \& Berger, B. Improving the power of GWAS and avoiding confounding from population stratification with PC-select. Genetics 197, 1045-1049 (2014).

68. Berner, D., Adams, D. C., Grandchamp, A. C. \& Hendry, A. P. Natural selection drives patterns of lake-stream divergence in stickleback foraging morphology. J. Evol. Biol. 21, $1653-1665$ (2008).

69. Blekhman, R. et al. Host genetic variation impacts microbiome composition across human body sites. Genome Biol. 16, 191 (2015).

70. Ghannoum, M. A. et al. Characterization of the Oral Fungal Microbiome (Mycobiome) in Healthy Individuals. PLoS Pathog. 6, e1000713 (2010).

Shan, X. \& Cordero, O. Deconstructing the association between abiotic factors and species assemblages in the global ocean microbiome. bioRxiv 2020.03.12.989426 (2020). doi:10.1101/2020.03.12.989426

72. Susin, A., Wang, Y., Lê Cao, K.-A. \& Calle, M. L. Variable selection in microbiome compositional data analysis. NAR Genomics Bioinforma. 2, (2020).

73. Pawlowsky-Glahn, V. \& Buccianti, A. Compositional Data Analysis Theory and Applications Edited by. (2011).

74. Aitchison, J. Principles of compositional data analysis. in 73-81 (Institute of Mathematical Statistics, 1994). doi:10.1214/1nms/1215463786

75. Asgari, E., Garakani, K., Mchardy, A. C. \& Mofrad, M. R. K. MicroPheno: predicting environments and host phenotypes from 16S rRNA gene sequencing using a k-mer based representation of shallow sub-samples. doi:10.1093/bioinformatics/bty296

76. Skums, P. et al. Efficient error correction for next-generation sequencing of viral amplicons. BMC Bioinformatics 13 Suppl 10, S6 (2012).

768 77. Martin, J. et al. Rnnotator: An automated de novo transcriptome assembly pipeline from stranded RNA-Seq reads. BMC Genomics 11, 663 (2010).

78. Carvalho, A. B., Dupim, E. G. \& Goldstein, G. Improved assembly of noisy long reads by

772 79. Garud, N. R. \& Pollard, K. S. Population Genetics in the Human Microbiome. Trends in Genetics 36, 53-67 (2020).

774 80. Wang, J. \& Jia, H. Metagenome-wide association studies: Fine-mining the microbiome. 
Nature Reviews Microbiology 14, 508-522 (2016).

776 81. Chen, P. E. \& Shapiro, B. J. The advent of genome-wide association studies for bacteria. Current Opinion in Microbiology 25, 17-24 (2015).

82. Zeller, G. et al. Potential of fecal microbiota for early-stage detection of colorectal cancer. Mol. Syst. Biol. 10, 766 (2014).

83. Pasolli, E., Truong, D. T., Malik, F., Waldron, L. \& Segata, N. Machine Learning Metaanalysis of Large Metagenomic Datasets: Tools and Biological Insights. PLOS Comput. Biol. 12, e1004977 (2016).

84. Truong, D. T. et al. MetaPhlAn2 for enhanced metagenomic taxonomic profiling. Nature Methods 12, 902-903 (2015).

85. LaPierre, N., Ju, C. J. T., Zhou, G. \& Wang, W. MetaPheno: A critical evaluation of deep learning and machine learning in metagenome-based disease prediction. Methods 166, 7482 (2019).

86. Baran, Y. \& Halperin, E. Joint analysis of multiple metagenomic samples. PLoS Comput. Biol. 8, (2012).

87. Rahman, M. A. \& Rangwala, H. RegMIL: Phenotype Classification from Metagenomic

88. Asgari, E., Garakani, K., McHardy, A. C. \& Mofrad, M. R. K. MicroPheno: predicting environments and host phenotypes from 16S rRNA gene sequencing using a k-mer based representation of shallow sub-samples. Bioinformatics 34, i32-i42 (2018).

89. Edwards, R. A., McNair, K., Faust, K., Raes, J. \& Dutilh, B. E. Computational approaches to predict bacteriophage-host relationships. FEMS Microbiol. Rev. 40, 258-272 (2016).

799 90. Marçais, G. \& Kingsford, C. A fast, lock-free approach for efficient parallel counting of $800 \quad$ occurrences of k-mers. Bioinformatics 27, 764-770 (2011).

801 91. Hoffman, G. E. \& Schadt, E. E. variancePartition: Interpreting drivers of variation in complex gene expression studies. BMC Bioinformatics 17, 483 (2016).

803 92. Pedregosa, F. et al. Scikit-learn: Machine Learning in Python. J. Mach. Learn. Res. 12, $804 \quad 2825-2830(2011)$.

805 93. Loomba, R. et al. Gut Microbiome-Based Metagenomic Signature for Non-invasive 
808 94. Qin, J. et al. A human gut microbial gene catalogue established by metagenomic

809 sequencing. Nature 464, 59-65 (2010).

810

811

812

813

814

815

816

817

818

819

820

821

822

823

824

825

826

827

828

829

830

831

832

833

834

835 